\title{
Primera cita y confirmación de reproducción de Gomphus vulgatissimus (Linnaeus, 1758) (Odonata: Gomphidae) en Gipuzkoa (País Vasco, España).
}

\section{First record and confirmation of reproduction of Gomphus vulgatissimus (Linnaeus, 1758) (Odonata: Gomphidae) in Gipuzkoa (Basque Country, Spain).}

Leire Paz ${ }^{1}$, Miguel A. Conesa²

*

\section{Resumen}

Con la detección en 2016 de una larva de Gomphus vulgatisimus (Linnaeus, 1758) en el río Araxes se cita la especie por primera vez en el Territorio Histórico de Gipuzkoa (N Península Ibérica), confirmándose asimismo su reproducción en este enclave.

Palabras clave: Odonata, Gomphus vulgatissimus, larva, reproducción, Gipuzkoa, España.

\begin{abstract}
Following the discovery in 2016 of a Gomphus vulgatissimus larva (Linnaeus, 1758) in the river Araxes, this species is recorded for the first time in the province of Gipuzkoa (N Iberian Peninsula), confirming its reproduction on this site.
\end{abstract}

Key words: Odonata, Gomphus vulgatissimus, larvae, reproduction, Gipuzkoa, Spain.

\section{Laburpena}

2016an Araxes ibaian Gomphus vulgatisimus (Linnaeus, 1758) odonatuaren larba bat aurkitzean, espezie hau lehen aldiz aipatzen da Gipuzkoa Herrialdean (Iberiar Penintsulako iparraldea), eta berresten da aldi berean beraren ugalketa kokaleku horretan.

Gako hitzak: odonatua, Gomphus vulgatisimus, larba, ugalketa, Gipuzkoa, Espainia.

\footnotetext{
${ }^{1}$ EKOLUR Asesoría Ambiental SLL • Camino de Astigarraga 2, PI. 4a dcha.-Of. 8. • 20180 Oiartzun

*Correspondencia: leire@ekolur.com
}

${ }^{2}$ Asociación Odonatológica de Andalucía

*Correspondencia: mconesa@libelulas.org 
Gomphus vulgatisimus (Linnaeus, 1758) es una especie de libélula común en gran parte de Europa, desde Francia a Bulgaria y sur de Finlandia (mapa en Boudot \& Kalkman, 2015); sin embargo, en la Península Ibérica no es frecuente y consecuencia de ello, son las pocas referencias bibliográficas existentes: en Noain y en Ancín en Navarra (Eizaguirre, 1988; Rabina \& Llamas, 2011), en el río Baias y en el Ega en Álava (Ocharan \& Ocharan, 2002; Gainzarain et al., 2013), en el río Duero en Soria (Salvador et al., 2014), en el río Ebro en Cantabria (Prunier et al. 2015), en las inmediaciones del río Pisuerga en Palencia (Salvador et al., 2014), en Astorga y en el río Cea en León (Ocharan, 1984; Belle, 1985; Ocharan Larrondo et al., 2011; Torralba-Burrial \& Ocharan, 2013; Conesa \& Bernal, 2013) y en Gándaras de Budiño en Pontevedra (Jödicke, 1996) (Fig. 1). Además, en la plataforma de Biodiversidad Virtual existe una fotografía de una hembra de la especie tomada en 2011 al sur de Bizkaia, aunque la localización no ha sido validada y recogida en la revisión de Prunier et al. 2015.

Debido a la escasez de citas, aparentemente fragmentadas y distantes del resto del área de distribución -es muy rara en el sur de Francia (Grand \& Boudot, 2006)-, este anisóptero ha sido incluido en el Libro Rojo de especies amenazadas de España con la categoría UICN de "vulnerable", criterio UICN B2ab(iii) (Ocharan Larrondo et al., 2011) y, a nivel de la Comunidad Autónoma del País Vasco, en el Catálogo Vasco de Especies Amenazadas, en la categoría de "vulnerable".

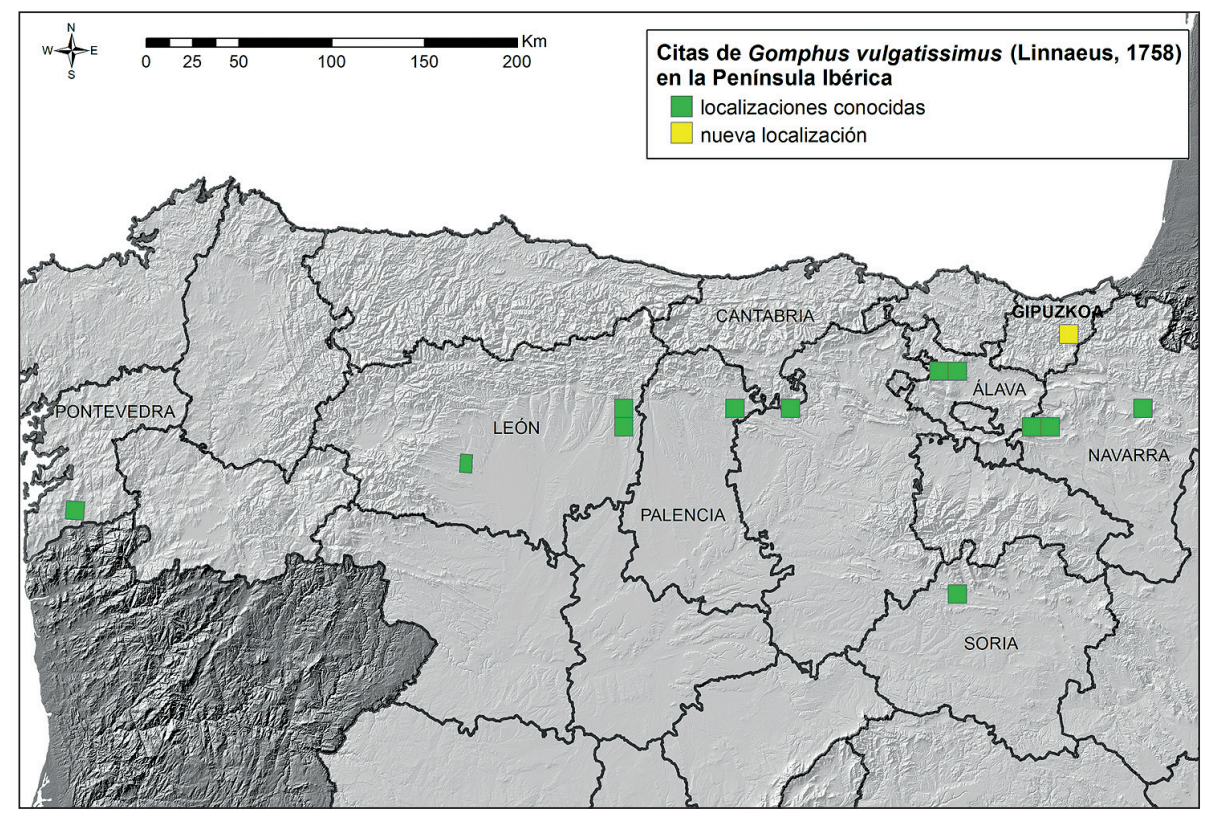

Fig. 1.- Situación de la nueva cita de Gomphus vulgatissimus (Linnaeus, 1758) respecto a las citas previas de la especie en la Península Ibérica.

Fig. 1.- Location of the new record of Gomphus vulgatissimus (Linnaeus, 1758) with regard to previous records of this species on the Iberian Peninsula. 
En el curso de los trabajos de seguimientos piscícolas contratados por la Diputación Foral de Gipuzkoa, se capturó mediante manga acuática una larva F0 de la especie G. vulgatissimus a mediados de septiembre de 2016 en el río Araxes (unidad hidrológica del Oria), situado en la parte oriental del Territorio Histórico de Gipuzkoa y cuyo nacimiento se ubica en Navarra. El punto en concreto se localiza en Lizartza (30TWN7870), a una altitud de 150 msnm, dentro del tramo designado como Zona Especial de Conservación (ZEC ES2120012 Araxes Ibaia/Río Araxes), en el cual la citada Administración llevó a cabo en 2011 un proyecto de mejora del cauce mediante aporte de madera muerta.

En concordancia con el hábitat descrito en la literatura (Heidemann \& Seidenbusch, 2002), la larva se encontraba enterrada en una zona de acumulación de arenas, sedimentos finos y detritus, cercana a la orilla, a una profundidad aproximada de medio metro bajo el agua y en un tramo de $8 \mathrm{~m}$ de anchura y con vegetación de ribera de tipo aliseda ribereña eurosiberiana. El ejemplar fue lavado, identificado in situ según las claves de Heidemann \& Seidenbusch (2002), fotografiado (Fig. 2) y devuelto en el punto de captura.

Otras especies de odonatos que se identificaron también en fase de larva en el tramo fueron: Calopteryx virgo (Linnaeus, 1758), Boyeria irene (Fonscolombe, 1838) y Onychogomphus uncatus (Charpentier, 1840).

Esta cita constituye, por tanto, la primera prueba de la presencia y reproducción de la especie en la provincia de Gipuzkoa y, apunta a que también pueda estar presente en las localidades limítrofes en Navarra (Fig.1). Con la adición de G. vulgatissimus, la fauna conocida de odonatos de Gipuzkoa asciende a 45 especies (Mezquita-Aranburu \& Ocharan, 2013; Mezquita-Aranburu, 2016).

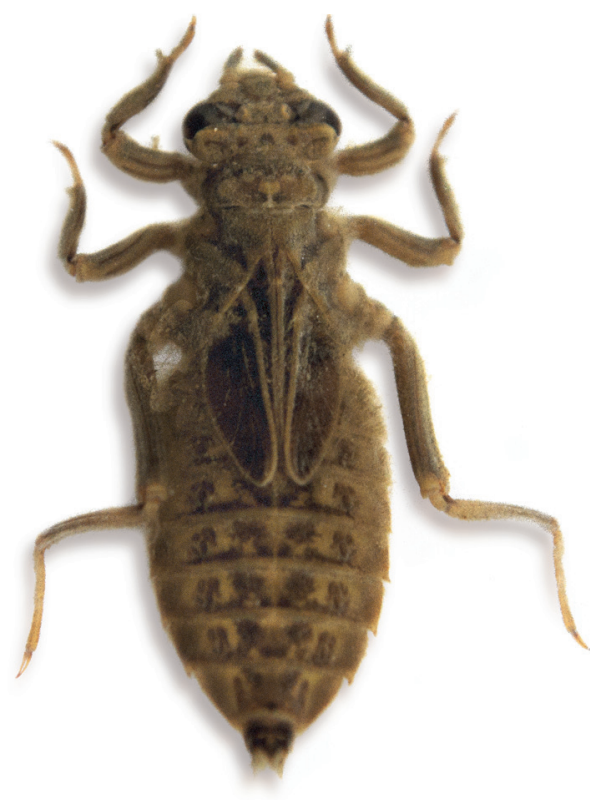

Fig.2.- Ejemplar de Gomphus vulgatissimus (Linnaeus, 1758) localizado en el río Araxes (Gipuzkoa).

Fig. 2.- Gomphus vulgatissimus specimen (Linnaeus, 1758) found in the river Araxes (Gipuzkoa). 


\section{Bibliografía}

- Belle, J. 1985. Gomphus vulgatissimus (Linnaeus) new to the fauna of Spain, with records of other interesting Odonata Anisoptera. Entomol. Ber. 45: 14-15.

- Boudot, J.-P., Kalkman, V.J. (Eds.) 2015. Atlas of the European dragonflies and damselflies. KNNV publishing, th Netherlands.

- Conesa, M.A., Bernal, A. 2013. Sobre la larva de Gomphus vulgatissimus (Linnaeus, 1758). Bol. S.O.A. 1: 7-8.

- Eizaguirre, S. 1988. Confirmación de un nuevo odonato para la Península Ibérica. Munibe, Cienc. Nat. 40: 125-129.

- Grand, D., Boudot, J.P. 2006. Les libellules de France, Belgique et Luxembourg. Biotope, Metz.

- Heidemann, H., Seidenbusch, R. 2002. Larves et exuvies des libellules de France et d'Allemagne (sauf de Corse). Société Française d'Odonatologie, Bois d'Arcy.

- Jödicke, R. 1996. Faunistic data of dragonflies from Spain. Adv. Odonatol., Suppl. 1: 155-189.

- Mezquita-Aranburu, I. 2016. Primera cita de Sympetrum sanguineum (Müller, 1764) (Odonata, Libellulidae) para Gipuzkoa (País Vasco, España). Munibe, Cienc. Nat.,64: 161-165.

- Mezquita-Aranburu, I., Ocharan, F.J. 2013 Odonatos de Gipuzkoa. Munibe, Cienc. Nat, 60: 51-75.

- Ocharan, F.J. 1984. Captura de Gomphus vulgatissimus (L.) en el Norte de España (Odonata; Gomphidae). Bol. Cien. Nat. Inst. Estud. Astur. I.D.E.A. 34: 3-6.

- Ocharan Larrondo, F.J., Torralba-Burrial, A., Outomuro Priede, D., Ocharan Ibarra, R., Cordero Rivera, A., Azpilicueta Amorín, M. 2011. Gomphus vulgatissimus (Linnaeus, 1758). En: Atlas y Libro Rojo de los invertebrados amenazados de España. Verdú, J.R., Nums, C. \& Galante, E. (Ed.): 569-573. Ministerio de Medio Ambiente y Medio Rural y Marino.

- Ocharan, R., Ocharan, F.J. 2002. Odonatos del Valle de Cuartango (Álava). Bol. Asoc. Esp. Entomol. 26: 97-110.

- Prunier, F., Brotóns, M., Cabana, M., Campos, F., Casanueva, P., Chelmick, D., Cordero Rivera, A., Díaz Martínez, C., Evangelio, J.M., Gainzarain, J.A., García-Moreno, J., Lockwood, M., de los Reyes, L., Mañani, J., Mezquita-Aranburu, I., Muddeman, J., Ocharan, F.J., Otero Pérez, F., Prieto Lillo, E., Requena, C., Ripoll, J., Rodríguez Luque, F., Rodríguez, P., Romeo, A., Salcedo, J., Salvador Vilariño, V., Sánchez Balibrea, J., Tamajón Gómez, R, Torralba Burrial, A., Tovar, C., Winter, P., Zaldívar, R. 2015. Actualización del inventario provincial de Odonatos de España peninsular e Islas Baleares. Bol. ROLA 6: 59-84.

- Rabina, E., Llamas, A. 2011. Nueva cita de la libélula Gomphus vulgatissimus L., 1758 para la Península Ibérica en Ancín, Navarra. Munibe, Cienc. Nat. 59: 77-80.

- Salvador, V., Flechoso, M.F., Rojo, I. 2014. Ampliación de la distribución conocida de odonatos amenazados en Castilla y León (España). Bol. S.E.A. 55: 279-287.

- Torralba-Burrial, A., Ocharan, F.J. 2013. Iberian Odonata distribution: data of the BOS Arthropod Collection (University of Oviedo, Spain). ZooKeys 306: 37-58.

- Fecha de recepción/Date of reception: 02-01-2017

- Fecha de aceptación/Date of acceptance: 10-03-2017

Editor Asociado/Associate editor: Alberto Castro 